\title{
The injectosome of bacteriophage P22
}

L. Tang, ${ }^{*}$ G. Lander,* S.R. Casjens, ${ }^{* *}$ W.R. Marion***, G. Cingolani*, P.E. Prevelige Jr. $* * *$ and J.E. Johnson*

* Department of Molecular Biology, The Scripps Research Institute, 10550 North Torrey Pines Road, La Jolla, CA 92037

$\S$ Department of Molecular Biosciences, University of Kansas, 1200 Sunnyside Ave., Lawrence, KS 66045

** Division of Cell Biology and Immunology, Department of Pathology, University of Utah Medical School, Salt Lake City, UT 84112

*** Department of Microbiology, University of Alabama at Birmingham, BBRB 416/6, 845 19th St. South, Birmingham, AL 35294

Double-stranded DNA (dsDNA) bacteriophage has been thought to be the most abundant biological entity on this planet [1]. The ultra-high efficiency of phage infection is partially attributed to a tail structure in most dsDNA bacteriophage such as P22 and T4. The tail is a multi-component molecular machine situated at a unique vertex of the phage capsid, and directs various critical biological events during phage infection such as host recognition and attachment, membrane penetration, and DNA translocation. E. coli Phage T4 contains a contractile long tail, a very complex macromolecular assembly made up of more than 20 proteins each present in multiple copies [2]. This $1200 \AA$ long tail appears to utilize the mechanical force created by the contraction of the tail sheath to drill a hole in the host cell membrane, through which the phage DNA is released into the host cytoplasm. In contrast, Salmonella phage P22 represents a distinct category of bacteriophage that contains a short, non-contractile tail. The P22 tail contains only 5 types of proteins, and yet displays full capability to deliver phage DNA from within the capsid into host cytoplasm in cooperation with several phage accessory proteins. Hence, the tail and the accessory proteins of P22 form an elegant injectosome that injects phage DNA into the host cell. We investigated the three-dimensional structures of the tail of the P22 injectosome in both isolated and in situ forms by means of electron cryo-microscopy (cryoEM). The EM data of the isolated P22 tail were collected by the tilt method as particles showed preferential orientation on EM grids. The cryoEM structure of the P22 tail was determined using the single particle reconstruction approach, and revealed a molecular machine consisting of the dodecameric portal, the hexa-trimeric tailspike, the dodecameric or hexameric gp4 and gp10, and the trimeric gp26 [3]. The cryoEM structure of the whole P22 virion illuminated the molecular interactions among the tail proteins, the capsid, and the phage DNA (Figure 1 and 2). These structures provided novel insights into the infection of bacteriophage P22 [4].

\section{References}

[1] H. Brussow et al., Cell 108 (2002) 13.

[2] M.G. Rossmann et al., Curr Opin Struct Biol 14 (2004) 171.

[3] L. Tang et al., EMBO J 24 (2005) 2087.

[4] The research in LT's laboratory is supported by a fund from University of Kansas. This work was supported by an NIH grant to JEJ. 


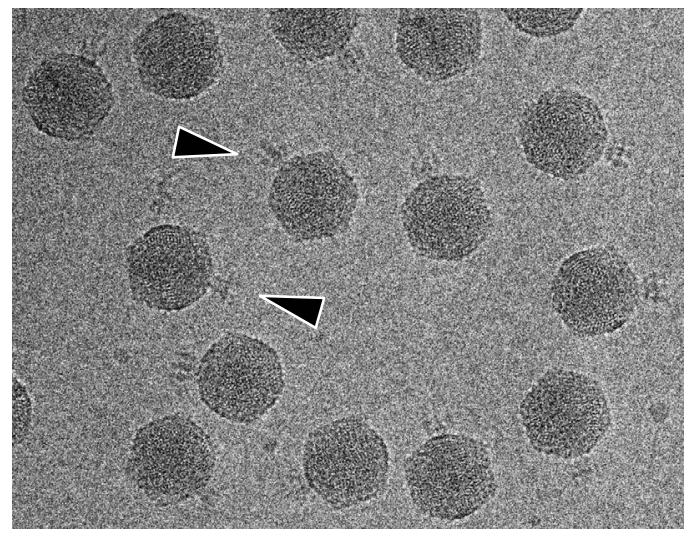

FIG. 1. Electron micrograph of frozen-hydrated phage P22. Arrowheads, the tail structure of phage P22.

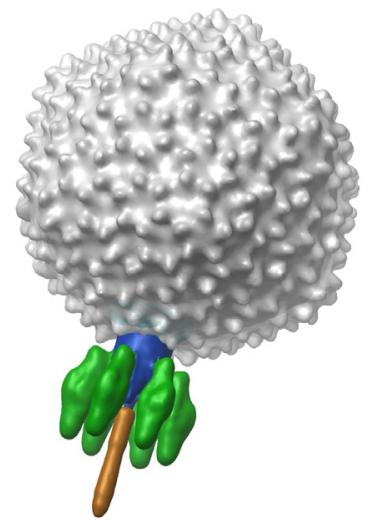

FIG. 2. The model of the P22 virion. The cryoEM structures of the isolated tail (color isosurface) and the P22 capsid (gray isosurface) are superimposed based on the reconstruction of the $\mathrm{P} 22$ virion. 